\title{
Exploration of Web front-end development technology and optimization direction
}

\author{
Cuiyu Fu \\ Department of software engineering, Hainan College of Software Technology, Qionghai \\ Hainan ,571400, China
}

Key words: Web front-end development technology, Optimization technology, Analysis.

\begin{abstract}
. under the background of rapid development of network technology, network field gains certain expansion. Thus, more and more people seriously depend on network in life and work. Meanwhile, most projects or jobs also enhance the degree of network dependence. It thus can be seen that network technology development plays an important role un social progress. Information presentation of Web front-end development technology in network operation process exerts a key function. It can not just influence user experience and application, but also bring influence on enterprise development. Thus, optimization of Web front-end development technology has important practical significance. This paper analyzes and studies Web front-end development technology, expounds its working property and proposes the optimization direction of this technology so as to better improve Web front-end development technology and improve user experience.
\end{abstract}

\section{Introduction}

In current information environment, the influence of internet on social production and life deepens continuously. Even in special environment, activity implementation needs complete dependence on network. Web front-end development technology plays an important role in presenting webpage and always bears the responsibility of network environment information representation. Actual working circumstance will have direct influence on internet users who experience information transmission and enjoy services, and even bring certain influence on achievement of information environment value and economic activity implementation in information environment. Thus, comprehensive optimization of Web front-end development technology has positive promotion function in terms of improving internet use experience. Therefore, it is necessary to value and focus on optimization of this technology and promotes its sustainable development.

\section{Overview of Web front-end development technology}

Web front-end development technology is a process of first easiness and then difficulty. It mainly includes three elements: HTML, CSS and JavaScript. Besides, the development personnel should master basic knowledge of network property optimization, SEP an server side, be familiar with application of various tools for auxiliary development, code maintenance, hierarchical semantic template and browser classification support etc. For website development and design personnel, all-round improvement of user experience and active attraction of users to visit have become very important working contents. Thus, in the process of Web front-end development technology, sound presentation of webpage content should be thought to make sure users can gain more experience. Next, this paper states and analyzes Web front-end development technology in detail.

\section{HTML, CSS and JavaScript}

In Web front-end development technology, HTML, CSS and JavaScript play a crucial role. The three kinds of languages undertake different tasks in the development project. Besides, they also have certain difference in operation method and standards ${ }^{[1]}$. However, the three languages have close connection. Rational application of the three can complete project development. The structure and pattern of HTML are relatively ideal. In actual application process, HTML can replace Flash 
gradually. CSS is the rule of controlling webpage appearance. The application of this language can continuously enhance webpage appearance effect 9mainly including shadow and transparency). In this way, it can coincide with webpage variety demand in modern society and better attract users to visit. The major function of JavaScript is to help users acquire the optimal picture effect and make the code and content separate.

\section{SEO}

SEO aims to completely adjust and optimize internal and external of website after natural ranking mechanism of search engines is known comprehensively. Then, natural ranking of key words in website search engine can improve to gain more ideal presentation quantity, make sure more users to click and visit the website and finally achieve the purpose of network marketing and brand building. SEO optimization can be divided into eight steps: firstly, analyze key words and especially analyze the quantity of key words concerned, competitors or network correlation; secondly, analyze website architecture. If the preferences of website architecture and search engine crawler coincide, optimization effect can be enhanced; thirdly, optimize website catalogue and page, make sure website homepage is in the ideal ranking of search engines and then make various pages of website being traffic; fourthly, content release and link arrangement; fifthly, dialogue with search engine; sixthly, actively construct website map SiteMap; seventhly, improve the quality of friendly link; eighthly, analyze website traffic in detail.

Webpage loading speed will generate direct influence on page view of users and consumers. The major reason is that people increase the requirements for the speed and efficiency of surfing the internet as modern life pace accelerates. The practice survey result shows that even if webpage loading time changes subtly, actual page view, trading volume and volume of business will be influenced. The improvement of traditional webpage loading speed will usually take into account of hardware condition improvement or network speed improvement. However, under the background of rapid development of science and technology, the application of explosive mobile Web browser achieves breakthrough of traditional improvement mode.

\section{Browser compatibility problem}

In the process of Web front-end development, there are a great variety of browsers. Besides, different browsers will have different analysis approaches and results for the same segment of code. Finally, different page display effects will form. Thus, to effectively avoid the above problems, it is necessary to apply multiple forms of auxiliary tools to test the bugs of different browsers in Web front-end development technology. Generally speaking, it is necessary to analyze attribute and built-in style of main browsers in Web front-end development, deeply know common bug handling methods and ensure development pertinence ${ }^{[2]}$. If possible, browser compatibility test should be conducted.

\section{Nature of Web front-end technology work}

To better optimize Web front-end technology, and gain long-term development, relevant workers should know and master relevant details involved in Web front-end work. Only when the above contents are known comprehensively can all-round optimization of Web front end be achieved. Meanwhile, optimization effect can be enhanced.

In current network environment, B/S structure is usually adopted. Thus, work emphasis should focus on analysis of user browser. This is also the method which is closest to current internet network environment features. When users acquire information, server page request is needed in the browser, and the website that the user wants to visit should be inputted. DNS server is used to analyze the domain inputted. Finally, the user enters webpage to meet user visit demand. On this basis, user browser will sends out a request to HTTP of server. After the server receives the request, it will confirm corresponding file information content according to specific request. But, current Web server usually uses relevant files in database in accordance with corresponding content and URL parameter and then meets the demand of different users ${ }^{[3]}$. 
After the file of HTML form is generated by the browser, the server will execute the request the user proposes to the browser, transmit effective file of HTML form to the browser used, and receive effective file in the server. After the above operation is completed, user' s much time will be consumed no matter whether the user inputs website or DNS request. Technical personnel optimize Web front end to improve user experience satisfaction degree. So, the key point of optimization work is the process from user' $s$ website input to final acquisition of the information required.

\section{Optimization direction of Web front-end development technology}

In the process of optimizing Web front end, the requirement for technology is quite high, and certain convenience will be brought for relevant users. However, in actual development work, the importance of Web front-tend technology optimization is easily ignored. However, Web front-tend technology optimization is very crucial in terms of improving property and promoting efficiency. Optimization of relevant technology can shorten webpage loading speed, and make timely responses to various operations so as to enhance experience effect. In addition, Web front end optimization can reduce the probability of rest request to the server, decrease bandwidth occupancy and achieves effective resource saving and efficiency promotion ${ }^{[4]}$.

\section{HTML page optimization}

Under the precondition of ensuring basic property, visual effect needs to be effectively improved in terms of website or webpage design. The major reason is that visual effect may be easily concerned by experiencers. Simply speaking, the pictures or animation can attract more experiencers to achieve enhancement of experience effect. But in practice, if a large batch of pictures is loaded at the same time, webpage loading speed will be affected, and loading time will extend. Actual survey shows that the increase in loading time will seriously influence the number of webpage users and even result in the loss of many users. Thus, technical personnel need to consider loading the picture content in the first screen during opening the webpage, and take full advantage of the first screen for introduction so as to better attract users for deep understanding. Other pictures can be loaded as required by the rolling the take full advantage of. In this way, page loading speed can improve effectively.

On this basis, it is required to effectively prevent gorgeous or tedious phenomenon during designing website page. This will not just result in aesthetic fatigue, but also generate adverse impacts on actual loading speed. During designing webpage, practicability should be mainly considered. Meanwhile, it is required to ensure page conciseness and explicit theme so that webpage operation efficiency can improve.

\section{Reduction of HTTP request}

In webpage design process, designers and relevant technical personnel will pay attention to the functions of picture and script and attract experiencers through processing pictures. But in actual application process, there are many strip links in the page. Thus, page loading speed slows down. For different webpage contents, it is required to make sure the function of script is exerted normally and try to make the page more concise. Unnecessary gorgeous pictures should not be used. This will not just generate adverse influence on actual visit speed, but also result in page skip or redirection operation phenomenon.

\section{Proper reduction of file size}

In terms of webpage loading speed and broadband resource occupancy, file size will be greatly influenced. Thus, it is required to make the best of relevant technology or delete unnecessary codes to effectively reduce file size. In order to improve webpage loading speed, the part which occupy the resources should be deleted. JavaScript or CSS file may be used to compress and reduce file size ${ }^{[5]}$. Besides, it is necessary to study and improve programming thought. In other words, relatively mature technical means and approaches should be fully applied to reduce code content. The most obvious 
manifestation is to eliminate unnecessary HTML label, optimize CSS or avoid inline style and finally make code file diminish.

\section{reduction of DNS inquiry}

In the process of inquiring DNS, the analysis process needs long time. Before the inquiry ends, relevant website will not load any content under the domain of the website. It thus can be seen that, if DNS inquiry is relatively complex and tedious, loading time will increase, finally influence user experience effect and restrict further development of website. Thus, DNS inquiry time should be reduced properly. Only in this way, page loading speed can improve.

\section{Prevention of redirection problem}

The emergence of redirection problem will cause consumers or users wait for long time. Redirection will increase request times of page for Web front end. For this problem, technical personnel needs to add "/" behind Web catalogue so as to effectively prevent redirection problem.

\section{Conclusion}

In conclusion, humans society gradually enters digital era in the process of social technological development. Besides, the dependence of poplar' $s$ work and life on network and computer technology also boosts gradually. Thus, it is necessary to improve Web front-end development technology. The optimization of Web front-end technology can bring certain influence on user experience. Therefore, operators gradually attach great importance to this problem. But for the above problem, targeted optimization and improvement are required. Technical personnel should continuously learn professional knowledge in professional knowledge, accumulate practical experience according to specific conditions and improve their professional skills. Meanwhile, page working state should be observed carefully, and the analysis should be conducted from multiple perspectives. Only in this way, Web front-end technology optimization can be achieved, and actual use effect can be enhanced. Webpage loading speed can be promoted through overall optimization of Web front-end development technology. Meanwhile, user experience effect can improve and work efficiency can improve. Moreover, unnecessary resource occupancy can be relieved so that resource utilization ratio and utilization value can improve greatly,

\section{References}

[1] Chen Yue, Qin Fujian, Exploration of Web Web technology and optimization direction, China Computer \& Communication, 2016(4):35,37.

[2] Chen Fei, Analysis of Web front-end development technology and optimization direction, New Media Research, 2015(7):39-40.

[3] Wang Zheng, Study on Web front-end development technology and optimization, Computer Knowledge and Technology, 2013(22):5037-5038.

[4] Zhao Dawei, Chen Gang, Study on talent training mode of Web front-end development technology, Computer Knowledge and Technology, 2015,11(24):109-110.

[5] Liu Xiaodong, Study on Web Front-end Development Technology teaching reform, Fujian Computer, 2016,32(4):77-77,88. 